\title{
A Network Pharmacology Study to Explore Mechanism of the Drug Pair of Astragalus-Saposhnikoviae Radix in the Treatment of Allergic Rhinitis
}

\author{
Chen LU, Limin MA, Haozhen WANG, Xiuting HUANG, Xiujin ZHANG, Ziyin LU \\ and Xiuli $\mathrm{LU}^{1}$ \\ Department of Biochemistry and Cell Biology, School of Life Science, Liaoning \\ University, China
}

\begin{abstract}
Allergic rhinitis (AR) has now become one of the major diseases affecting people's lives, and Traditional Chinese medicine (TCM) always has good efficacy in clinical treatment. In the present study, we analyzed the most frequently used drug pair of Astragalus-Saposhnikoviae Radix (SR) in prescriptions for the treatment of allergic rhinitis by network pharmacology to reveal the modern pharmacological mechanisms of drug prevention and treatment of the disease. Firstly, the 38 active ingredients with good ADME properties from the Astragalus-SR drug pair were collected from the database, and the collated drug targets of Astragalus and SR and the targets of allergic rhinitis were mapped against each other by the network visualization software Cytoscape, followed by the establishment of a "drug active ingredient-target-disease" network diagram and the construction of a highconfidence protein-protein interaction network. Then, the common targets obtained from the disease and drug active ingredients were imported by $\mathrm{R}$ language for GO enrichment analysis and KEGG pathway enrichment analysis. The KEGG pathways associated with the targets of Astragalus and SR for the treatment of allergic rhinitis obtained from $\mathrm{R}$ enrichment analysis were imported into Cytoscape, and the CytoNCA plug-in was loaded to construct a "target-pathway" network map, and the core target wogonin (FN1) was screened. These evidences suggest that the drug pair of Astragalus-SR works in a multi-component, multi-target and integrated modulation manner for the treatment of allergic rhinitis, which provides an important basis for the treatment of allergic rhinitis.
\end{abstract}

Keywords. Allergic rhinitis, traditional Chinese medicine, network pharmacology, Astragalus, Saposhnikoviae Radix (SR)

\section{Introduction}

In recent years, the incidence of allergic rhinitis is on the rise worldwide [1]. The prevalence in Western countries has reached $10 \%-20 \%$, and in some countries even $30 \%$,

\footnotetext{
${ }^{1}$ Corresponding author, Xiuli LU, Department of Biochemistry and Cell Biology, School of Life Science, Liaoning University, China; Email: luxiuli@lnu.edu.cn.
} 
while in China it is $8.5 \%-21.3 \%$ [2, 3]. In the traditional classification, allergic rhinitis is divided into seasonal allergic rhinitis and perennial allergic rhinitis. About $20 \%$ of them are seasonal allergic rhinitis, $40 \%$ are perennial allergic rhinitis, and the remaining $40 \%$ are perennial allergic rhinitis combined with seasonal episodes. In 2001 the World Health Organization made a new classification of allergic rhinitis based on its onset, its impact on the patient's quality of life, and the course of the disease. The onset of the disease was identified as intermittent if it occurred less than four days per week or for a duration of fewer than 28 days, and as persistent if it occurred more than four days per week or for a duration of more than 28 days. In terms of quality of life, normal sleep, daily activities, physical recreation, and work and study are considered mild, while one or more of these are not normal and are called moderate to severe allergic rhinitis [4].

It is generally believed that allergic rhinitis is induced by deficiency of the lung, spleen, kidney, or by chill, as well as by foreign body attacked [4]. In ancient China, the etiology of allergic rhinitis was no more than two major aspects: heat syndrome and cold syndrome, repletion pattern, and deficiency syndrome $[5,6]$.

Modern studies have shown that allergic rhinitis is a histopathological reaction of the respiratory tract of an atopic individual after stimulation by antigenic substances. The stimulated nasal and bronchial mucosa has a large number of Th2 cytokine-expressing helper $T$ cells, eosinophils, and mast cell infiltrates involved in the metaplastic disease of the nasal mucosa, along with the release of inflammatory mediators such as histaminemediated by IgE, triggering a similar inflammatory process [3].

The treatment of allergic rhinitis in TCM is mainly divided into the syndrome of cold fluid retention in lung (CFRL), Spleen Qi deficiency syndrome, kidney Yang deficiency syndrome, blood-stasis and internal obstruction syndrome, and lung heat fumigation syndrome $[4,7]$. By reviewing the literature and statistics, it was found that Astragalus and Saposhnikoviae Radix (SR) are the single herbs with the highest frequency in the formulas for the treatment of allergic rhinitis, and the drug pair of Astragalus and SR is the most often used combination in treatment of AR with significant therapeutic effects [7].

Saposhnikoviae Radix (SR) is a perennial herb that belongs to the family Umbelliferae, which mostly grows in northeast, northwest, and north China. It has multifunction including antioxidant, antipyretic and analgesic, anti-inflammatory, and antitumor effects. In the treatment of allergic rhinitis, it has been found that it could inhibit IgE in animals which plays an important role in the onset and persistence of allergic rhinitis, thus preventing the release of inflammatory factors and therefore relieving the inflammatory response. In addition to this antiallergic, antiviral, and immune-enhancing properties of Astragalus [8-11].

Astragalus is a perennial herb of the family Legume. It mostly grows in northeast, northwest, and north China. It has the efficacy of tonifying the Qi and fixing the surface, diuretic and toxin, drainage of pus, and astringency of sores and muscle. According to the Chinese herbal medicine chemical composition database, in addition to some trace elements, Astragalus includes 68 effective chemical components such as Astragalus polysaccharide, Astragaloside, Astragalus and flavonoids, which can enhance the immunity of the body, improve blood flow changes and other pharmacological effects, as well as dual regulation of immune function [12-15]. 
Network pharmacology is the cross-fertilization of biology and computer science, through the cross-analysis of all the active ingredients in prescriptions and disease targets, the visualization network construction of related nodes, the systematic and comprehensive expression of drug intervention and influence on the disease network, revealing the mystery of multi-molecular drug synergy in the human body [16], which is consistent with the theory of Chinese medicine to diagnose and treat diseases from a holistic perspective, prescription multi-component. This is in line with the theory of Chinese medicine to diagnose and treat diseases from a holistic perspective, and the principle of multi-component, multi-pathway and multi-target synergistic action of prescriptions, which undoubtedly points the way to the modernization and internationalization of Chinese medicine.

In this study, we focused on the network pharmacology study of Astragalus-SR drug pair in the treatment of allergic rhinitis, and predicted the targets of the drug for the disease treatment of allergic rhinitis by analyzing the results of network pharmacology studies, the possible involvement of active ingredients in the regulation of cell metabolism and signal transduction. Our data revealed the scientific basis for the use of Astragalus-SR drug pair in the treatment of allergic rhinitis through drug pair, and also provides a basis for exploring the use of traditional Chinese medicine for the treatment of other diseases.

\section{Materials and Methods}

\subsection{Acquisition and Collation of Drug Targets in TCMSP}

The TCMSP (Traditional Chinese Medicine Systems Pharmacology Database and Analysis Platform, https://www.tcmsp-e.com/) was used to collect the active ingredients of Astragalus and SR for the treatment of allergic rhinitis with oral bioavailability (OB $\geq 30 \%$ ) and drug-likeness ( $\mathrm{DL} \geq 0.18$ ) as the screening requirements for the eligible components and relevant targets. OB is an important indicator to evaluate a drug's efficacy [17]. DL is the similarity between a given ingredient and its known equivalent in Western medicine. Later, the active ingredients that did not appear in the database or had strong activity but low OB or DL values were collated by reading the literature and grouped to analyze whether they were used as study subjects.

\subsection{ID Conversion of Drug Targets}

The data obtained by TCSMP needs to be standardized before subsequent operations can be carried out. We used UniProt (https://www.uniprot.org/) to download all the data, and compared the data obtained by TCSMP with the data obtained by UniProt in Excel, then used vlookup function to further improve the data through data comparison and filling, and finally completed rapid and accurate standardization through screening. 


\subsection{Acquisition of disease targets}

GeneCards (https://www.genecards.org/) can quickly find information about human genes and is used to collect critical information at the initial stage of the user's gene research, saving a lot of time [18]. We used the GeneCard and the DisGeNET (https://www.disgenet.org/) to search and collect allergic rhinitis-related target genes using "Allergic rhinitis" as a keyword to indicate allergic rhinitis, and the collected related disease targets were processed, integrated, and de-redundant targets to obtain Allergic rhinitis-related targets were processed, integrated and de-redundant targets were obtained and saved in text format.

\subsection{Building a Venn Diagram of Disease Targets and Component Targets}

SwissTargetPrediction (http://www.swisstargetprediction.ch/) can be used for similar and directly homologous species, mapping predictions by homology within and between different species, and is a powerful tool for screening drug small molecule compound targets [19]. We combined, de-duplicated and collated the drug targets obtained from TCSMP with SwissTargetPrediction to obtain drug targets and determined the number of targets, and the data obtained from DisGeNET database was collated by de-duplication to obtain disease targets and determined the number of targets. Then, the drug active ingredient targets were intersected with the disease targets to determine the number of overlapping targets, and a Wayne diagram was drawn using $\mathrm{R}$ language which was used to process data. In this experiment, we installed the clusterProfiler, org.Hs.eg.db, enrichplot and ggplot2 packages, and then imported the data for processing and analysis [20].

\subsection{KEGG and GO Enrichment Analysis and "Drug-Component-Target" Network Construction}

GO functional enrichment analysis with KEGG pathway enrichment analysis using R language can help us to gain insight into differentially expressed genes and the enrichment relationships between them at the molecular level to find GO taxonomic entries and KEGG pathways that are enriched for differential genes. In this experiment to further obtain key targets, Cytoscape (https://cytoscape.org/) was used to install the plugin BisoGenet, importing common targets of drug pairs and diseases into this database and limiting the species to human species (Homo sapiens, Human), and finally obtaining through serial screening a highly confident of PPI and core network maps [21]. The processed data can then be imported into Cytoscape using the Cytoscape software installation plugin CytoNCA to obtain the enriched relationships of genes with related pathways and export the visual images for data analysis. The shape and size of the graph representing the data can be adjusted by Style to visualize the relationship between targets and pathways.

In this experiment, we performed GO enrichment analysis and KEGG pathway enrichment analysis on the potential targets of Astragalus and SR drugs on active ingredients for the treatment of allergic rhinitis and draw bubble and histogram to analyze the output results. Cytoscape 3.7.2 can also be used to visualize the signaling pathways 
and the targets involved in the KEGG pathway enrichment analysis, i.e., to build a "target-pathway" network.

\section{Results}

\subsection{Active Components of Drug Targets and Action Targets of AR}

The 87 active components of Astragalus and 173 active components of SR were obtained through the TCMSP and literature reading, totaling 260 active ingredients. Then, 20 Astragalus and 18 SR were obtained by screening with Oral Bioavailability $(\mathrm{OB}) \geq 30 \%$ and Drug-likeness $(\mathrm{DL}) \geq 0.18$, totaling 38 potentially active compounds with good ADME properties. The chemical information of each active ingredient was tabulated by number, molecular name, $\mathrm{OB}$ and $\mathrm{DL}$ to construct a dataset of active ingredient information of Astragalus and SR. By comparing Astragalus and SR no active ingredients were duplicated, therefore, a total of 38 active ingredients were obtained.

\subsection{AR Targets}

The GeneCards database and the DisGeNET database were used to search and collect allergic rhinitis-related target genes using "Allergic rhinitis" as the keyword, and the collected disease targets were processed and integrated. Redundant targets were removed, and tabulations were organized and saved in the text file format. A total of 98 allergic rhinitis targets were collected.

\subsection{Statistical Results of Disease-Component Interaction Targets}

The drug target of 38 effective compounds of Astragalus and SR obtained through TCSMP were searched through the two databases of TCSMP and SwissTargetprediction, and a total of 389 drug targets were collected after standardization and de-redundancy of bioDBnet software.

The disease target genes related to allergic rhinitis were obtained through the GeneCards database and DisGeNET database. After sorting and de-redundancy of the collected related disease targets, 446 related targets of allergic rhinitis were obtained. 48 overlap targets were obtained by the intersection of disease targets and drug targets. The Venn diagrams were drawn by installing VennDiagram package using $\mathrm{R}$ language, as shown in figure 1. 


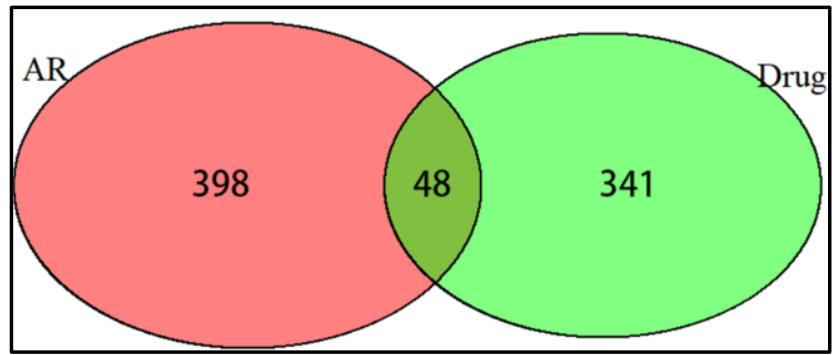

Figure 1. Venn diagram of drug-disease shared targets of action. Note, the red part is the target of allergic rhinitis; the green part is the target of Astragalus and SR drugs; intersecting parts are common targets.

\subsection{Active Ingredient-Target-Allergic Rhinitis Network Construction and Analysis}

The "active ingredient-target-allergic rhinitis" network graph was constructed using Cytoscape 7.2 and network graph analysis was performed. The results showed that the network diagram contains 81 nodes (33 compounds and 48 potential targets) (figure 2). Table 1 provides the main results of this analysis. In the network diagram, the node degree indicates the number of edges in the network that are connected to the node. For example, the connecting lines of each inverted triangle indicate the number of targets possessed by the Astragalus and SR active ingredients; while the connecting lines of each rectangle represent the number of Astragalus and SR active ingredients associated with the corresponding targets. The results showed that a total of 33 active ingredients from Astragalus and SR interacted with 48 potential targets and thus influenced the development of allergic rhinitis. These results suggest that Astragalus-SR drug pair act in a multi-target, multi-chemical, and integrative regulatory manner for the treatment of allergic rhinitis.

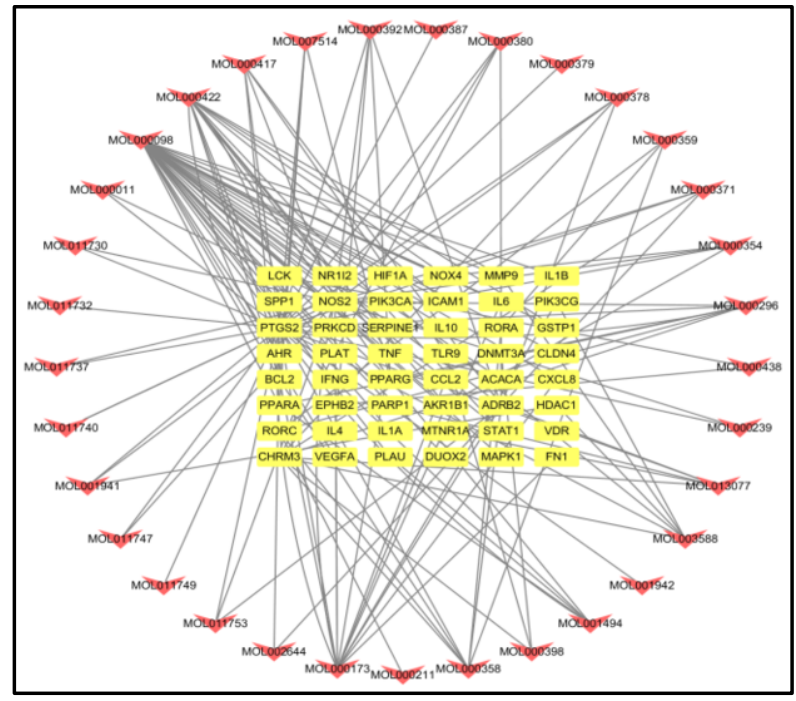

Figure 2. Component-target-disease interaction network diagram. Note, the yellow rectangles part is the target protein; the red inverted triangles part is the relevant active ingredient. 
Table 1. Main relevant target protein node degrees.

\begin{tabular}{llllll}
\hline Number & Target & Degree & Number & Target & Degree \\
\hline 1 & PTGS2 & 32 & 8 & PPARA & 5 \\
2 & NOS2 & 18 & 9 & AKR1B1 & 4 \\
3 & ADRB2 & 11 & 10 & AHR & 3 \\
4 & CHRM3 & 8 & 11 & MMP9 & 3 \\
5 & PPARG & 6 & 12 & NOX4 & 3 \\
6 & RORC & 6 & 13 & PIK3CA & 3 \\
7 & BCL2 & 5 & 14 & TNF & 3 \\
\hline
\end{tabular}

\subsection{PPI Network Key Target Genes Results}

To screen the core targets of Astragalus and SR sand for the treatment of allergic rhinitis, the Bisogenet functional package was loaded in Cytoscape 3.7.2 software, and the information of 48 potential targets was imported, and the database was set to ProteinProtein Interaction to obtain high-confidence PPI network information of the active ingredients and disease intersection targets of Astragalus and SR (figure 3). Based on this PPI network in Cytoscape software, the network topology was analyzed by using CytoNCA toolkit, which was calculated and tabulated in table 2. According to the relevant topological parameters, firstly, the network was filtered by the minimum value of Degree centrality (DC) 61 of the protein interactions network, removing the connections with duplicates between nodes, saving the filtered images, and create subnetworks; after that, the network centrality was calculated according to the intermediary number centrality (BC) of 288.3, the proximity centrality (CC) of 0.5509 , the eigenvector centrality (EC) of 0.068326 , the network centrality (Network Centrality, NC) is 33.76 in order to perform core target screening and create subnetworks and draw the network diagram (figure 4). Figure 4 shows the network topology analysis. After screening, the results showed that the Yellow node was the related gene of the core target protein, and the red node FN1 was the core target protein. The topological parameters of these targets meet the above card values and can be used as the core targets of Astragalus and SR in the treatment of allergic rhinitis [22].

Table 2. Top 10 of core target topology parameters of Astragalus-SR drug pair for the treatment of allergic rhinitis.

\begin{tabular}{|c|c|c|c|c|c|c|}
\hline Number & Gene symbol & Degree (DC) & $\begin{array}{l}\text { Betweenness } \\
\text { centrality (BC) }\end{array}$ & $\begin{array}{l}\text { Closeness } \\
\text { Centrality } \\
\text { (CC) }\end{array}$ & $\begin{array}{l}\text { Eigenvector } \\
\text { Centrality } \\
\text { (EC) }\end{array}$ & $\begin{array}{l}\text { Network } \\
\text { Centrality } \\
\text { (NC) }\end{array}$ \\
\hline 1 & NPM1 & 359 & 5133.241 & 0.647255 & 0.125746 & 65.56716 \\
\hline 2 & MCM2 & 518 & 8767.207 & 0.653782 & 0.1259 & 59.0005 \\
\hline 3 & RPS8 & 156 & 535.4493 & 0.569546 & 0.089517 & 55.83243 \\
\hline 4 & RPL7A & 136 & 311.407 & 0.560519 & 0.084225 & 54.32419 \\
\hline 5 & RPLP0 & 157 & 579.8889 & 0.56623 & 0.087929 & 53.76952 \\
\hline 6 & RPS4X & 154 & 409.0875 & 0.56623 & 0.085118 & 51.61799 \\
\hline 7 & RPS3A & 155 & 689.7889 & 0.570381 & 0.08601 & 51.25897 \\
\hline 8 & RPL6 & 161 & 480.3851 & 0.569546 & 0.086736 & 51.10189 \\
\hline 9 & RPS6 & 153 & 427.342 & 0.562139 & 0.081935 & 50.60122 \\
\hline 10 & RPL7 & 143 & 457.2453 & 0.567883 & 0.083389 & 49.47102 \\
\hline
\end{tabular}




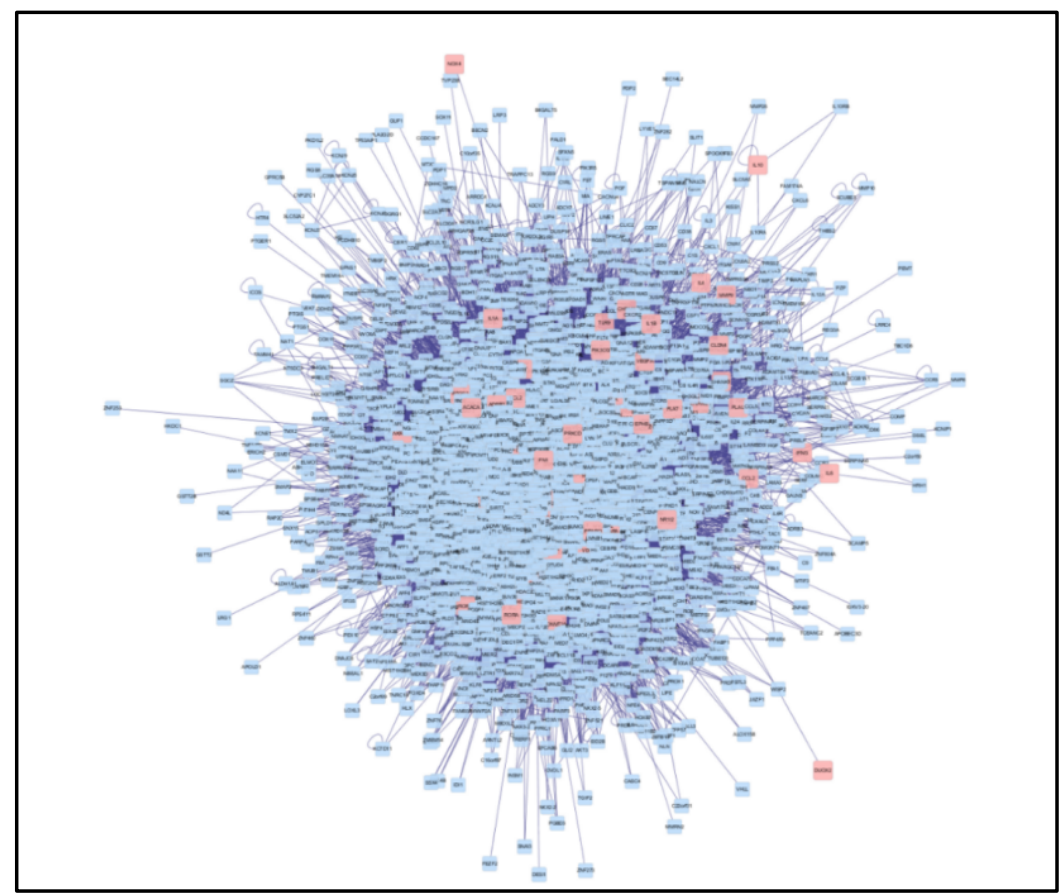

Figure 3. High confidence interaction network diagram (PPI) of target proteins of Astragalus and SR for the treatment of allergic rhinitis.

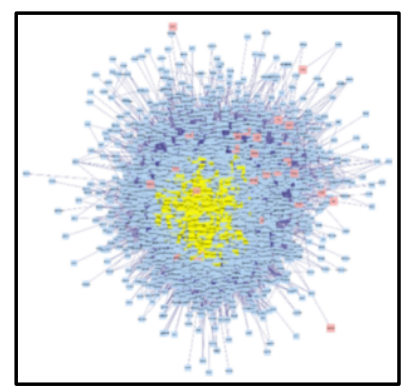

(a)

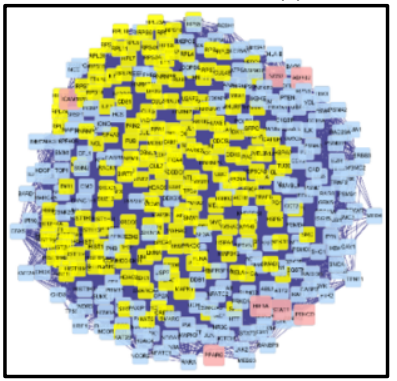

(c)

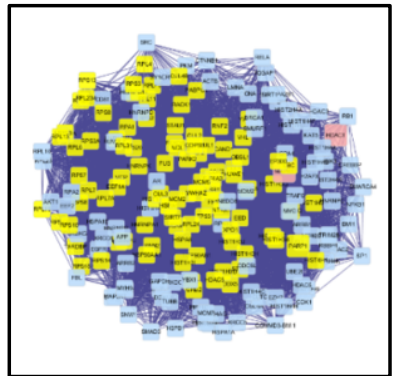

(d)

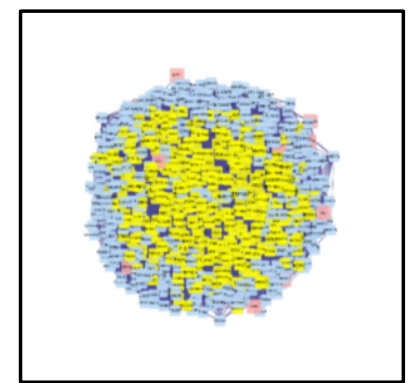

(b)

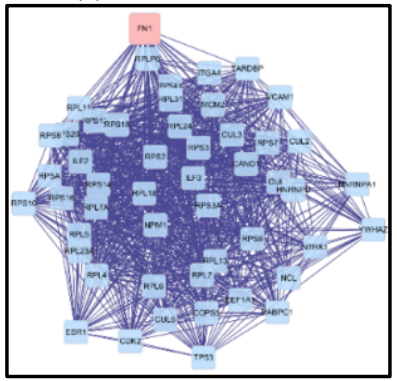

(e)

Figure 4. Core target map for topology analysis of the target network of Astragalus and Fructus for the treatment of allergic rhinitis. Note (1), red is input target gene; yellow is input target-associated protein gene. Note (2), (a) is DC screen; (b) is BC screen; (c) is CC screen; (d) is EC screen; (e) is NC screen. 


\subsection{Results and Analysis of KEGG Enrichment Analysis}

In order to illustrate the role of the active components of drug pair of Astragalus and SR in the signal pathway of allergic rhinitis, the KEGG enrichment analysis in R language was used to analyze the 48 potential targets in the above "active component target disease" network diagram. Through the parameter $\mathrm{p}$ value $(\mathrm{P}<0.01)$ and false discovery rate, FDR $<0.5$ ) we determined $20 \mathrm{KEGG}$ entries and drew corresponding histogram and a bubble chart, which were shown in table 3 and figure 5 . According to table 3 , the active components of Astragalus-SR drug pair can affect the development of allergic rhinitis through signaling pathways such as leishmaniasis, tuberculosis, rubella and IL-17 signaling pathway.

Table 3. Potential target KEGG enrichment pathways by Astragalus-SR drug pair associated with allergic rhinitis.

\begin{tabular}{lllll}
\hline Number & Name of the passageway & $\boldsymbol{p}$ value & FDR & Count \\
\hline hsa05140 & Leishmaniasis & $1.31 \mathrm{E}-06$ & $7.75 \mathrm{E}-05$ & 6 \\
hsa05152 & Pulmonary tuberculosis & $1.40 \mathrm{E}-06$ & $7.75 \mathrm{E}-05$ & 8 \\
hsa05162 & Measles & $3.04 \mathrm{E}-06$ & 0.000106 & 7 \\
hsa04657 & IL-17 signaling pathway & $4.24 \mathrm{E}-06$ & 0.000106 & 6 \\
hsa05417 & Lipids and atherosclerosis & $5.30 \mathrm{E}-06$ & 0.000106 & 8 \\
hsa05142 & Chagas disease & $6.83 \mathrm{E}-06$ & 0.000106 & 6 \\
hsa04620 & TLRS signaling pathway & $7.64 \mathrm{E}-06$ & 0.000106 & 6 \\
hsa04625 & C-type lectin receptor signaling pathway & $7.64 \mathrm{E}-06$ & 0.000106 & 6 \\
hsa04659 & Th17 cell differentiation & $9.01 \mathrm{E}-06$ & 0.000111 & 6 \\
hsa05321 & Inflammatory bowel disease & $1.16 \mathrm{E}-05$ & 0.000114 & 5 \\
hsa05145 & Toxoplasmosis & $1.17 \mathrm{E}-05$ & 0.000114 & 6 \\
hsa05164 & Influenza A & $1.24 \mathrm{E}-05$ & 0.000114 & 7 \\
hsa04919 & Thyroid hormone signaling pathway & $1.83 \mathrm{E}-05$ & 0.000155 & 6 \\
hsa04080 & Neuroactive ligand receptor interaction & $2.05 \mathrm{E}-05$ & 0.000162 & 9 \\
hsa05143 & African trypanosomiasis & $2.43 \mathrm{E}-05$ & 0.000179 & 4 \\
hsa04210 & Apoptotic pathway & $3.55 \mathrm{E}-05$ & 0.000245 & 6 \\
hsa05022 & Neurodegeneration - multiple diseases & $4.64 \mathrm{E}-05$ & 0.000301 & 10 \\
hsa04932 & Nonalcoholic fatty liver & $6.15 \mathrm{E}-05$ & 0.000367 & 6 \\
hsa05222 & small-cell carcinoma of the lung & $6.31 \mathrm{E}-05$ & 0.000367 & 5 \\
hsa05163 & Human cytomegalovirus infection & $7.01 \mathrm{E}-05$ & 0.000387 & 7 \\
\hline
\end{tabular}

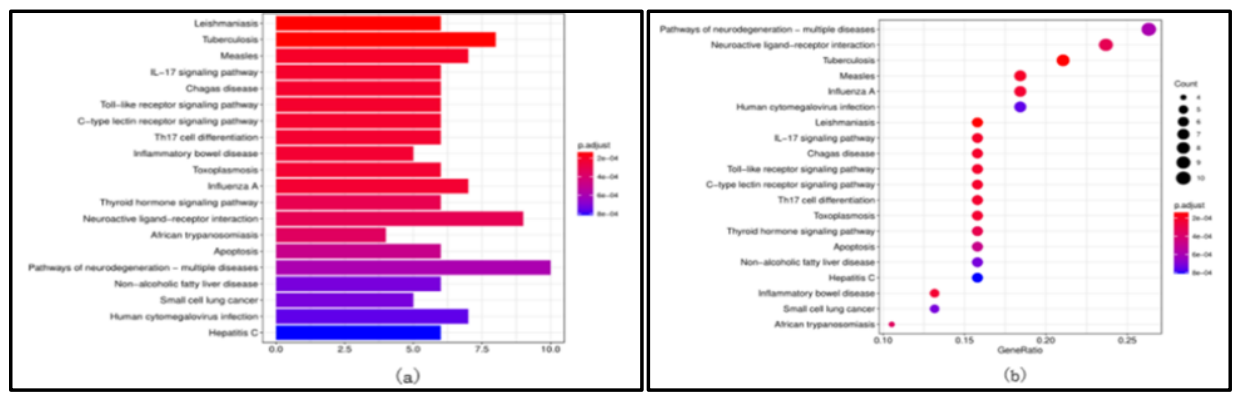

Figure 5. Histograms and bubble plots of KEGG enrichment analysis of Astragalus-SR drug pair. 


\subsection{GO Enrichment Analysis Results and Analysis}

GO enrichment analysis was performed using $\mathrm{R}$ language to investigate the functions of 48 target protein-related genes involved in the above "component-target-disease" network diagram, and 10 major GO entries were identified by two parameters, P-value $(\mathrm{P}<0.05)$ and false discovery rate $(\mathrm{FDR}<0.1)$ (table 4$)$, the corresponding bar and bubble plots were plotted in figure 6. These enrichment results indicated that Astragalus-SR drug pair exerted their therapeutic effects on allergic rhinitis by regulating, for example, biological processes such as cell membrane surface channel protein type delivery, and immunomodulatory factor expression.

Table 4. Major Allergic rhinitis-related GO entries of Astragalus-SR drug pair against potential targets.

\begin{tabular}{|c|c|c|c|c|c|}
\hline Category & $\begin{array}{l}\text { Term } \\
\text { identification } \\
\text { number }\end{array}$ & Functional & $P$ value & FDR & Count \\
\hline GOTERM_BP_DIRECT & GO:0019932 & $\begin{array}{l}\text { Second messenger mediated } \\
\text { signal }\end{array}$ & $7.45 \mathrm{E}-11$ & $5.18 \mathrm{E}-08$ & 12 \\
\hline GOTERM_BP_DIRECT & GO:0002237 & $\begin{array}{l}\text { Reaction to molecules of } \\
\text { bacterial origin }\end{array}$ & $9.68 \mathrm{E}-11$ & $5.18 \mathrm{E}-08$ & 11 \\
\hline GOTERM_BP_DIRECT & GO:0031649 & Fever & $1.76 \mathrm{E}-10$ & $6.27 \mathrm{E}-08$ & 5 \\
\hline GOTERM_BP_DIRECT & GO:2001234 & $\begin{array}{l}\text { Negative regulation of } \\
\text { apoptosis signaling pathway }\end{array}$ & $8.79 \mathrm{E}-10$ & $2.28 \mathrm{E}-07$ & 9 \\
\hline GOTERM_BP_DIRECT & GO:0032496 & $\begin{array}{ll}\text { Response } & \text { to } \\
\text { lipopolysaccharide } & \end{array}$ & $1.07 \mathrm{E}-09$ & $2.28 \mathrm{E}-07$ & 10 \\
\hline GOTERM_BP_DIRECT & GO:2001237 & $\begin{array}{l}\text { Negative regulation of } \\
\text { exogenous apoptosis } \\
\text { signaling pathway }\end{array}$ & 2.05E-09 & $3.66 \mathrm{E}-07$ & 7 \\
\hline GOTERM_BP_DIRECT & GO:0001660 & Fever & $3.24 \mathrm{E}-09$ & 4.96E-07 & 4 \\
\hline GOTERM_BP_DIRECT & GO:0031652 & $\begin{array}{l}\text { Positive regulation of } \\
\text { heating }\end{array}$ & $5.09 \mathrm{E}-09$ & $6.81 \mathrm{E}-07$ & 4 \\
\hline GOTERM_BP_DIRECT & GO:1903409 & $\begin{array}{l}\text { Biosynthesis of reactive } \\
\text { oxygen species }\end{array}$ & 7.20E-09 & 8.57E-07 & 7 \\
\hline GOTERM_BP_DIRECT & GO:2001233 & $\begin{array}{l}\text { Regulation of apoptosis } \\
\text { signaling pathway }\end{array}$ & $8.18 \mathrm{E}-09$ & $8.75 \mathrm{E}-07$ & 10 \\
\hline
\end{tabular}

\subsection{KEGG Relationship Network Results and Analysis}

In order to illustrate the role of active components of Astragalus and SR in the signal pathway in the treatment of allergic rhinitis, we performed KEGG enrichment analysis on 48 potential targets, and imported the obtained analysis data into Cytoscape. We chose to use the first 20 pathways and draw the corresponding target pathway network diagram, as shown in figure 7 . It is proved that it involves the signal pathway of Leishmania disease, tuberculosis, measles, IL-17, lipids and atherosclerosis. 

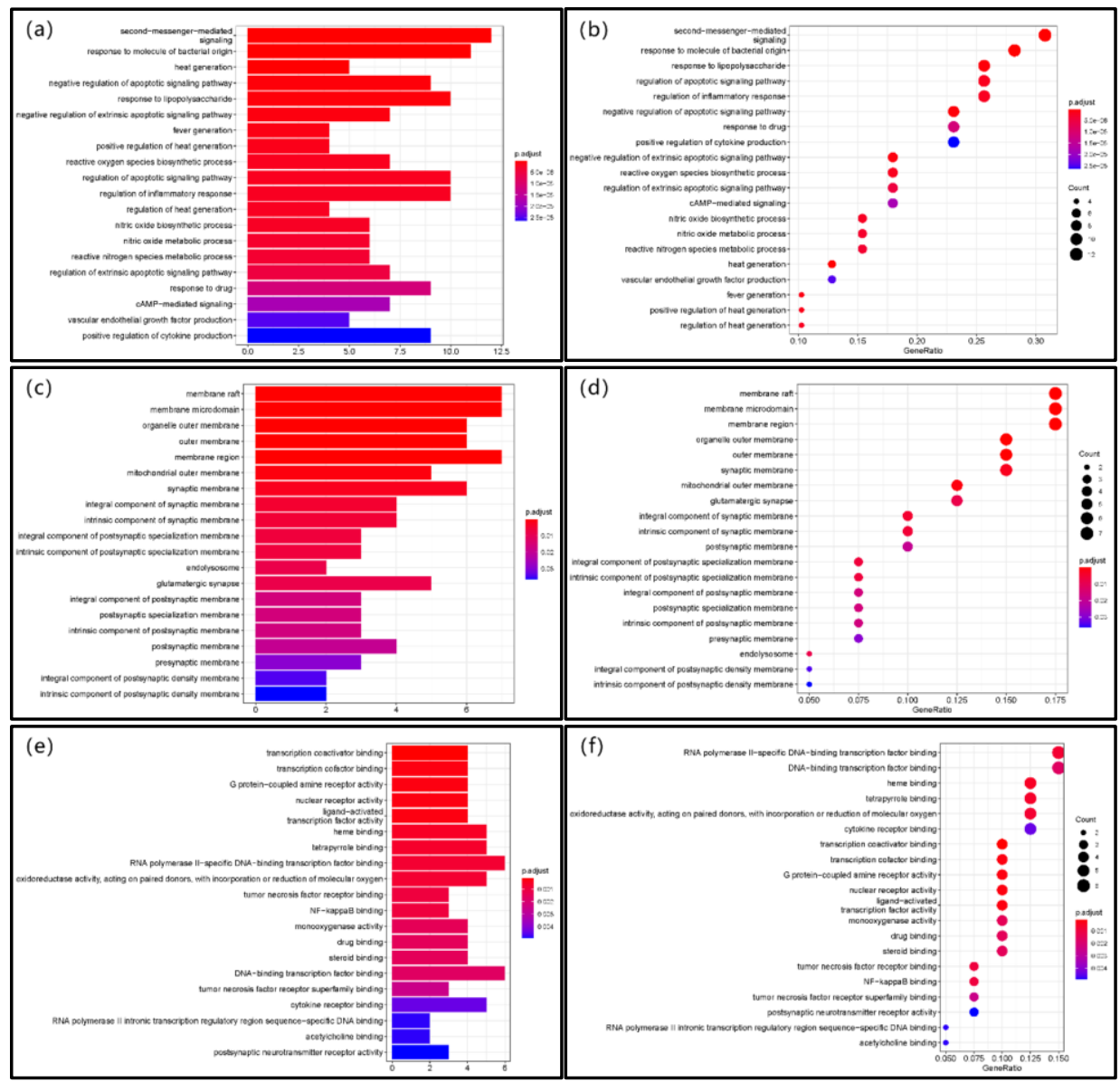

Figure 6. Histogram and bubble plot of GO enrichment analysis of potential targets by Astragalus-SR drug pair associated with allergic rhinitis. Note, (a) and (b) are the biological process (BP) histogram and bubble plot, respectively; (c), (d) are the cellular component (CC) histogram and bubble diagram, respectively; (e), (f) are molecular function (MF) histograms and bubble plots. 


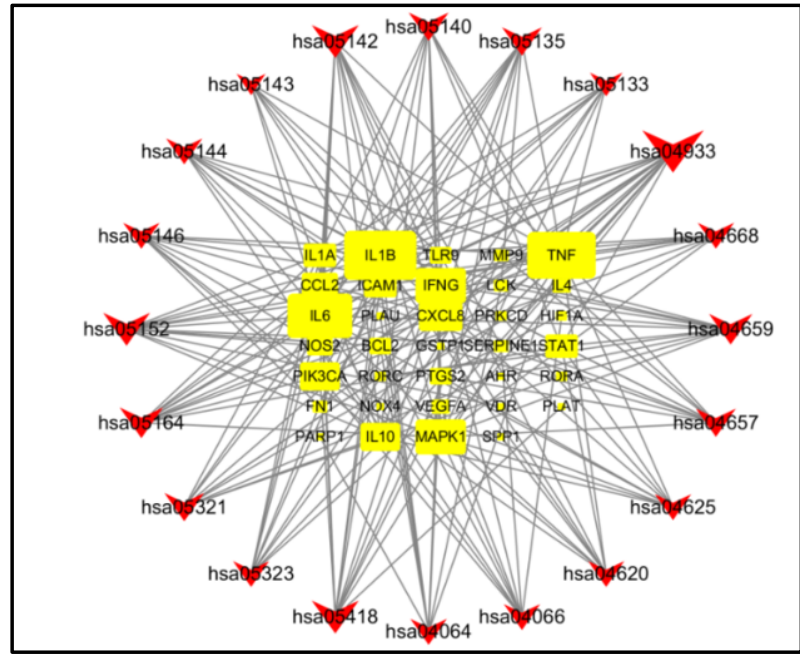

Figure 7. Target-pathway network diagram. Yellow is the target enriched to the signaling pathway; red is the signaling pathway.

\section{Discussion}

In this experiment, TCMSP, PharmMapper, Swiss TargetPrediction databases, Cytoscape and $\mathrm{R}$ language were used to analyze and screen drug active ingredients and drug-disease targets and to complete network mapping and analysis of the results. All the active chemical components related to Astragalus and SR drug pairs were collected from the TCMSP with Oral Bioavailability $(\mathrm{OB}) \geq 30 \%$ and Drug-Like $(\mathrm{OL}) \geq 0.18$ as the screening conditions for active ingredients and supplemented by literature review. The relevant targets of active ingredients were obtained after screening and de-duplication by TCMSP, PharmMapper and SwissTargetPrediction, and the targets were standardized by bioDBnet and other software. The target genes of allergic rhinitis were collected through Genecards, DisGeNET and other databases. We used Cytoscape to map the drug targets to the disease targets, and then mapped the "component-target-disease" network and performed PPI protein interaction network mapping, cytoNCA network topology analysis, GO function enrichment analysis, KEGG signaling pathway enrichment analysis, and KEGG relationship network construction. KEGG relationship network diagram was constructed. TCMSP database and literature reading and query obtained 87 active components of Astragalus and 173 active components of SR, a total of 260 active molecules. Later, 20 Astragalus and 18 SR were obtained by screening with $\mathrm{OB} \geq 30 \%$ and $\mathrm{DL} \geq 0.18$, totaling 38 potentially active compounds with good ADME properties. Allergic rhinitis-related target genes were collected using the GeneCards database and DisGeNET database, and the targets were processed and integrated to remove redundant targets, and a total of 98 allergic rhinitis targets were collected. PPI protein interaction network analysis showed that RELA, AR, HDAC3, KDR, PTPN1 and FN1 were potential targets for Astragalus and SR in the treatment of allergic rhinitis. The results of CytoNCA network topology analysis show that FN1 is the core target in the network. 
The FN1 gene encodes fibronectin, a glycoprotein formed by the cross-linking of two subunits through a disulfide bond formed at the C-terminus, and exists as a dimer or multimer on the cell surface and in the extracellular matrix. Fibronectin mediates cell adhesion and migration processes, including embryogenesis, wound healing, blood coagulation, host defense, and metastasis [23]. In the study of allergic rhinitis, FN1 is involved in abnormal immune responses of the body induced by environmental factors. In the rapid-onset phase of the response, it is involved in allergen stimulation to increase IgE-coated mast cells that recognize allergen degranulation to trigger symptoms. In the late-phase response phase, inflammatory mediators produced by participating mast cells act on vascular endothelial cells, causing them to express cell adhesion molecules and selectin E, leading to adhesion of leukocytes and vascular endothelial cells in the circulation and infiltration of nasal mucosal tissue by neutrophils, $\mathrm{T}$ cells and macrophages under the synergistic action of cytokines. Finally, the infiltrating inflammatory cells in the nasal mucosa release inflammatory mediators to cause symptoms [3].

The GO enrichment analysis of the 48 target protein-related genes involved in the above "component-target-disease" network showed that the biological process involves second messenger-mediated signaling, response to bacteria-derived molecules, and thermogenesis; the molecular function involves transcriptional co-activator binding, transcriptional cofactor binding, and $\mathrm{G}$ protein-coupled amine receptor activity; and the cellular components involve membrane rafts, membrane microdomains, organelle outer membranes, outer membranes, and membrane regions. The KEGG pathway enrichment analysis identified several signaling pathways and performed "target-pathway" network mapping, in which the signaling pathways involved leishmaniasis, tuberculosis, rubella, IL-17 signaling pathway, etc. The above results showed that Astragalus-SR drug pair are effective in the treatment of allergic rhinitis through a multi-component, multi-target and comprehensive regulation, which provides an important basis for the treatment of allergic rhinitis.

\section{Conclusion}

PPI protein interaction network analysis showed that RELA, AR, HDAC3, KDR, PTPN1 and FN1 were potential targets for Astragalus and SR in the treatment of allergic rhinitis. The results of CytoNCA network topology analysis show that FN1 is the core target in the network. The above results showed that Astragalus-SR drug pair are effective in the treatment of allergic rhinitis through a multi-component, multi-target and comprehensive regulation, which provides an important basis for the treatment of allergic rhinitis.

\section{Acknowledgments}

The study was supported by Liaoning provincial key R \& D project (No. 2020JH2/10300114 and 2019JH8/10300057). 


\section{References}

[1] DU GH. Principles of Pharmacology [M]. Renmin Weisheng Chubanshe(Chinese), May,2009.

[2] Wang XQ. Professor Wang Qingguo's academic thought and his characteristic experience in the treatment of allergic rhinitis with Shuangxin Bihe Decoction [J]. Beijing University of Chinese Medicine, 2015.

[3] Zhang L, Zhou B and Han DM, et al. Research progress of allergic rhinitis (I): pathogenesis [J]. ENT \&amp; HN Surgery Department, 2003, (05): 62-6+70.

[4] Huang SW. Ancient and modern literature research on the syndrome and treatment of allergic rhinitis. [D]; Beijing University of Chinese Medicine, 2006.

[5] Peng K. Syndrome differentiation and treatment of allergic rhinitis [J]. Guangming Zhongyi(Chinese), 2008, 23(11): 1798-9.

[6] Wang F. Study on TCM syndrome differentiation of allergic rhinitis in children [D]; Shandong University of traditional Chinese Medicine, 2009.

[7] Long YH. Study on medication law of traditional Chinese medicine in the treatment of allergic rhinitis based on literature retrieval [J]. Xiandai shangmao Gongye(Chinese), 2019, 000(009): 106-7.

[8] Chang FY,Chang G. Saposhnikoviae Radix is good at treating allergic rhinitis [J]. Zhongyi Zazhi(Chinese), 2003, 44(007): 493-493.

[9] Li YS, Xie H, Liu Y, et al. Study on the mechanism of Saposhnikoviae Radix-Magnolia in the treatment of allergic rhinitis based on network pharmacology [J]. Zhongyi Yanerbihou Zazhi(Chinese), 2020, 10(04): 185-90.

[10] Geng YM, Zhang ZW, Shi L. Effect of Saposhnikoviae Radix polysaccharide on immune factors in rats with allergic rhinitis [J]. Zhongguo Yishi, 2017, (7).

[11] Xiang F, Peng GY, Huang XA, Li LM, Qian PZ, Huang GL and Lin JL. Study on the mechanism of Yupingfeng Powder in the intervention of allergic rhinitis based on network pharmacology [A]. Chinese society of traditional Chinese medicine World Federation of traditional Chinese Medicine Societies. Proceedings of the 25th academic annual meeting of the otorhinolaryngology branch of the Chinese society of traditional Chinese medicine and the 11th academic annual meeting of the professional committee of Otorhinolaryngology and stomatology of the World Federation of Chinese medicine societies [C]. Chinese society of traditional Chinese medicine and World Federation of traditional Chinese Medicine Societies: otorhinolaryngology branch of the Chinese society of traditional Chinese medicine, 2019:2

[12] TONG X. Pharmacological effects of main active components of Astragalus. [J]. Shizhen Guoyi GUOyao(Chinese), 2011, 22(005): 1246-9.

[13] Zhang Q, Gao WY and Man SL. Chemical composition and pharmacological activities of Astragali Radix [J]. Zhongguo Zhongyao Zazhi, 2012, 037(021): 3203-7.

[14] Chen GH, Huang WF. Research Progress on chemical constituents and pharmacological effects of Astragalus membranaceus [J]. Zhongguo Xinyao Zazhi(Chinese), 2008, 17(017): 1482-5.

[15] Song YX. Clinical effect of Cangwu Tongqiao Powder on allergic rhinitis (deficiency of lung and qi) in children[D]; Changchun University of Chinese Medicine, 2019.

[16] PAN J H. New paradigm for drug discovery based on network pharmacology [J]. Chinese Journal of New Drugs and Clinical Remedies, .

[17] Liu J, Pei M, Zheng C, et al. A Systems-Pharmacology Analysis of Herbal Medicines Used in Health Improvement Treatment: Predicting Potential New Drugs and Targets. Evid Based Complement Alternat Med, 2013,2013(6):938764

[18] IOWA DNUO. GeneCards [J]. Springer Netherlands.

[19] ROLF A, AMOS B, WU C H, et al. UniProt: the Universal Protein knowledgebase [J]. Nucleic Acids Research. 32(Database issue): D115-9.

[20] Zhang J, Li MT. Bioinformatics analysis and design based on R software and database [J]. Xiandai Xinxi Keji(Chinese, 2020, v.4(04): 84-7.

[21] MARTIN A, OCHAGAVIA M E, RABASA L C, et al. BisoGenet: a new tool for gene network building, visualization and analysis [J]. BMC Bioinformatics, 2010, 11(1): 1-9.

[22] CytoNCA: A cytoscape plugin for centrality analysis and evaluation of protein interaction networks [J]. Biosystems, 2015, 127: 67-72.

[23] HGNC. https://www.ncbi.nlm.nih.gov/gene/2335 [Z]. 2016 